\title{
Solution of Dirac Equation for q-Deformed Eckart Potential with Yukawa-type Tensor Interaction for Spin and Pseudospin Symmetry Using Romanovski Polynomial
}

\author{
A. Suparmi ${ }^{*}$ and C. Cari \\ Physics Department, Post Graduate Study, Sebelas Maret University \\ Jl. Ir. Sutami no 36 A Kentingan, Surakarta 57126, Indonesia
}

\section{ARTICLE INFO \\ Article history: \\ Received 23 October 2013 \\ Accepted 10 November 2013 \\ Keywords: \\ Dirac equation solution \\ q-deformed eckart potential \\ Yukawa-type tensor \\ Spin symmetry \\ Pseudospin symmetry \\ Romanovski polynomials}

Received in revised form 02 November 2013

\begin{abstract}
A B S T R A C T
The motion of a nucleon in q-deformed Eckart potential field coupled with Yukawatype tensor potential is described by using Dirac equation. The bound state solutions of Dirac equation for q-deformed Eckart potential with Yukawa-type tensor potential under exact spin- and pseudospin-symmetric limit are obtained using finite Romanovski polynomials. The approximate relativistic energy spectra are exactly obtained within the approximation scheme of centrifugal term. The relativistic energy is negative for pseudospin symmetry and positive for spin symmetry. The radial component of Dirac spinors are obtained in terms of Romanovski polynomials under exact spin- and pseudospin-symmetric conditions. The relativistic energy spectrum for the exact spin-symmetric case reduces to nonrelativistic energy spectrum in the non-relativistic limit.
\end{abstract}

\section{INTRODUCTION}

The bound state solutions of Dirac equations for some central/non-central physical potentials, which are mixtures of attractive scalar potential $S(r)$, repulsive vector potential $V(r)$, and tensor interaction potential $U(r)$, have been intensively investigated since they have important applications in quantum chemistry and nuclear physics. Dirac equations are used to describe the motion of particles governed by strong force when relativistic effects are taken into account. Dirac equations for central/non-central potentials together with/without tensor potentials have been solved by NikiforovUvarov (NU) method [1-5], factorization method and supersymmetric quantum mechanics (Susy QM) [6], hypergeometric and confluent hypergeometric methods [7,8], and asymptotic iteration method [9]. Dirac equations with central/non-central potential are solvable exactly only for the s-wave For the l-wave, they are only solved approximately due to the contribution of the centrifugal term. The approximation scheme of the centrifugal term was proposed by Greene and Aldrich [10] and this

${ }^{*}$ Corresponding author.

E-mail address:suparmiuns@gmail.com approximation works well for hyperbolic/ exponential and trigonometric potentials.

Dirac equations with specific condition, where the substraction of the repulsive vector potential by an attractive scalar potential results in zero, have also been investigated recently. The potentials investigated include, among others, Makarov potentials [11,12], ring-shaped oscillator potentials [13], ring- shaped non-spherical harmonic oscillator potentials $[14,15]$, Coulomb potentials plus new ring-shaped potentials [16], and Hartmann potential plus new ring-shaped potential $[17,18]$. Those potentials are widely used in studying the relativistic effects of the distorted nucleus, the interaction between the ring-shaped molecules, and complex vibration-rotation energy structure of multi-electron atom.

The solution of Dirac equation with special cases of spin symmetry and pseudospin symmetry have been investigated for some typical potentials $[19,20]$. Spin-symmetric and pseudospin-symmetric concepts have been used to study the aspects of deformed and superdeformed nuclei in nuclear physics. The concept of spin symmetry has been applied to the spectra of meson and antinucleon [21], and the pseudospin symmetry concept is used to explain the quasi-degeneracy of nucleon doublets 
[22], exotic nuclei [23], and superdeformation in nuclei [24], and to establish an affective nuclear shell-model scheme [25]. Spin symmetry occurs when the repulsive vector potential subtracted by the attractive scalar potential is equal to a constant [26], while pseudospin symmetry arises when the sum of the scalar potential with vector potential is equal to a constant $[21,27]$.

Quantum deformation has been studied recently due to its relation with applications in nuclear physics [28], statistical-quantum theory and conformal field theory [29]. The q-deformation of hyperbolic potential, which was introduced by Arai [30], has been investigated by some authors [31,32]. Sauza Dutra [33] has reinterpreted the idea of q-deformed potential as a kind of parameter scaling symmetry of the model, so the q-deformed system is not a new class of potential. The q-deformed hyperbolic potential can be transformed to non-deformed hyperbolic potential or vice-versa by using translation of spatial variable.

In this paper, the relativistic energies and wave functions of q-deformed Eckart potentials with Yukawa-type tensor potentials [8] are analyzed using finite Romanovski polynomials. The Eckart potential is an anharmonic potential which is used to describe diatomic molecular energy spectra, electromagnetic transitions, and the internuclear potential in diatomic molecules [34,35]. The spherically symmetric Eckart-type potential is also used as molecular potential model which has been applied in chemical physics. The Yukawa potential, also known asthe screened Coulomb potential, was originally used to model strong nucleon-nucleon interactions caused by exchange interactions in nuclear physics and to explain the cloud of electronic charges around the nucleus [36,37]. The Yukawa potential was proposed to build the meson theory which describes nucleon-nucleon interactions $[38,39]$.

The finite Romanovski polynomial is a traditional method which consists of reducing Schrödinger equation by an appropriate change of variable to attain a form of generalized hypergeometric equation [40]. The polynomial was discovered by Sir E.J. Routh [41] and rediscovered 45 years later by V.I. Romanovski [42]. The term "finite" refers to the observation that for any given set of parameters (i.e. at any potential) only a finite number of polynomials appear orthogonal [43]. We apply the Romanovski polynomial method since this method is simpler than the NU method for obtaining the energy spectrum and the wave function, although there are limitations in determining the normalization of the wave function. Until recently, only few researcher used Romanovski polynomials to solve Schrödinger equation for certain potentials [44-46].

This paper is organized as follows. A brief review of the Dirac equation and the q-deformed potential are next presented in the second section. The finite Romanovski polynomials as an analysis method is presented in the third section. Solutions of Dirac equations, results, and discussions are presented in the fourth section. Finally, the last section presents a brief conclusion.

\section{THEORY}

\section{Basic equations of diracspinors}

A Dirac equation which describes the motion of a nucleon with mass $M$ in a combined potential which includes a repulsive vector potential $V(r)$, an attractive scalar potential $S(r)$, and a tensor potential $U(r)$ is given as $[8,47-49]$ :

$$
\begin{aligned}
& \{\vec{\alpha} \cdot \vec{p}+\beta(M+S(\vec{r}))-i \beta \alpha \cdot \vec{r} U(r)\} \psi(\vec{r}) \\
& =\{E-V(\vec{r})\} \psi(\vec{r})
\end{aligned}
$$

Where $E$ is the relativistic energy and $\vec{p}$ is the three dimensional momentum operator, $-i \nabla$,

$$
\vec{\alpha}=\left(\begin{array}{cc}
0 & \sigma \\
\sigma & 0
\end{array}\right), \text { and } \beta=\left(\begin{array}{cc}
I & 0 \\
0 & -I
\end{array}\right)
$$

where $\sigma$ is the three dimensional Pauli matrix, $I$ is the $2 \times 2$ identity matrix, and $U(r)$ is a tensor potential. Here we consider the matrix potential in equation (1) as a spherically symmetric potential; thus, they only depend on the radial coordinate $r=|\vec{r}|$ and we have taken $\hbar=1, c=1$. The Dirac equation expressed in equation (1) is invariant under spatial inversion, and the refore its eigenstates have definite parity.

The spinors are then written as

$$
\psi(\vec{r})=\left(\begin{array}{c}
\zeta(\vec{r}) \\
\varphi(\vec{r})
\end{array}\right)=\left(\begin{array}{c}
\frac{F_{n K}(r)}{r} Y_{j m}^{l}(\theta, \phi) \\
i \frac{G_{n K}(r)}{r} Y_{j m}^{\bar{l}}(\theta, \phi)
\end{array}\right)
$$

where $\zeta(\vec{r})$ is the upper (large) spinor component of the wave function and $\varphi(\vec{r})$ is the lower (small) spinor component, $\quad Y_{j m}^{l}(\theta, \phi)$ is $\quad$ spin-spherical harmonics, $\quad Y_{j m}^{\bar{l}}(\theta, \phi)$ is pseudospin-spherical harmonics, and $m$ is the projection of the angular 
momentum on the z-axis. The Hamiltonian of Dirac equation in a spherical field commutes with the total angular momentum operator $\vec{J}$ and the spin-orbit coupling operator $\vec{K}, K=-\beta(\vec{\sigma} \cdot \vec{L}+1)$ with $\vec{L}$ being the orbital angular momentum. The eigenvalues of the spin-orbit coupling operator are $\kappa=(J+1 / 2)>0$ for unaligned spin $\left(p_{1 / 2}, d_{3 / 2}, \ldots\right)$ and $\kappa=-(J+1 / 2)<0$ for aligned spin $\left(s_{1 / 2}, p_{3 / 2}, \ldots\right)$. Therefore the conservative quantities consist of the set of $\vec{H}, \vec{K}, \vec{J}, J_{z}$. Inserting equations (3) and (2) into equation (1), we obtain

$$
\begin{aligned}
& \left(\begin{array}{ll}
0 & \sigma \\
\sigma & 0
\end{array}\right) p\left(\begin{array}{l}
\zeta(r) \\
\phi(r)
\end{array}\right)+\left(\begin{array}{cc}
I & 0 \\
0 & -I
\end{array}\right)(M+S(\vec{r}))\left(\begin{array}{l}
\zeta(r) \\
\phi(r)
\end{array}\right)- \\
& i \beta \alpha . \bar{r} U(r)\left(\begin{array}{c}
\zeta(r) \\
\phi(r)
\end{array}\right)=(E-V(\vec{r}))\left(\begin{array}{c}
\zeta(r) \\
\phi(r)
\end{array}\right)
\end{aligned}
$$

From the matrices multiplication in equation (4) we obtain the upper and lower radial part of the Dirac equation, respectively, as

$$
\begin{aligned}
& \left\{\frac{d^{2}}{d r^{2}}-\frac{\kappa(\kappa+1)}{r^{2}}+\frac{2 \kappa}{r} U(r)-U^{2}(r)-\frac{d U}{d r}\right\} F_{n \kappa}(r) \\
& +\frac{d \Delta}{d r}\left(\frac{d}{d r}+\frac{\kappa}{r}-U(r)\right) /\left(M+E_{n \kappa}-\Delta(r)\right) F_{n \kappa}(r) \\
& +\left(M+E_{n \kappa}-\Delta(r)\right)\left(E_{n \kappa}-M-\Sigma(r)\right) F_{n \kappa}(r)=0
\end{aligned}
$$

And

$$
\begin{aligned}
& \left\{\frac{d^{2}}{d r^{2}}-\frac{\kappa(\kappa-1)}{r^{2}}+\frac{2 \kappa}{r} U(r)-U^{2}(r)+\frac{d U}{d r}\right\} G_{n \kappa}(r) \\
& +\frac{d \Sigma}{d r}\left(\frac{d}{d r}-\frac{\kappa}{r}+U(r)\right) /\left(M-E_{n \kappa}+\Sigma(r)\right) G_{n \kappa}(r) \\
& +\left(M+E_{n \kappa}-\Delta(r)\right)\left(E_{n \kappa}-M-\Sigma(r)\right) G_{n \kappa}(r)=0
\end{aligned}
$$

where the spin-orbit quantum number $\kappa$ is related to the usual orbital angular momentum by $\kappa(\kappa+1)=l(l+1)$ which corresponds to the upper spinor component and is related to pseudo-orbital angular momentum by $\kappa(\kappa-1)=\bar{l}(\bar{l}+1)$ for the lower spinor component, $\Sigma(r)=V(r)+S(r)$ is the sum of scalar and vector potentials, and $\Delta(r)=V(r)-S(r)$ is the vector potential subtracted by the scalar potential.

Pseudospin symmetry occurs when $\Sigma(r)=V(r)+S(r)=C_{p s}$ is constant, therefore the Dirac equation for the lower spinor in equation (6) reduces to

$$
\begin{aligned}
& \left\{\frac{d^{2}}{d r^{2}}-\frac{\kappa(\kappa-1)}{r^{2}}+\frac{2 \kappa}{r} U(r)-U^{2}(r)+\frac{d U}{d r}\right\} G_{n \kappa}(r) \\
& +\left(M+E_{n \kappa}-\Delta(r)\right)\left(E_{n \kappa}-M-C_{p s}\right) G_{n \kappa}(r)=0
\end{aligned}
$$

with $\kappa(\kappa-1)=\bar{l}(\bar{l}+1)$, where $\bar{l}_{\text {is }}$ the pseudoorbital angular quantum number $: \kappa=-\bar{l}$ for $\kappa<0 \quad$ and $\kappa=(\bar{l}+1) \quad$ for $\kappa>0 . \quad$ Also, $\bar{l}=l-\kappa /|\kappa|$. These conditions imply that the total angular momentum $j=\bar{l} \pm \frac{1}{2}$ is degenerate for $\bar{l} \neq 0[50,51]$.

Further, the exact pseudospin symmetry arises when $\Sigma(r)=V(r)+S(r)=C_{p s}=0, \quad$ and the q-deformed Eckart potential expressed in equation (10) is $\Delta(r)=V(r)-S(r)$ Therefore, equation (7) becomes

$$
\begin{aligned}
& \left\{\frac{d^{2}}{d r^{2}}-\frac{\kappa(\kappa-1)}{r^{2}}+\frac{2 \kappa}{r} U(r)-U^{2}(r)+\frac{d U}{d r}\right\} G_{n \kappa}(r) \\
& +\left(E_{n \kappa}{ }^{2}-M^{2}\right) G_{n \kappa}(r)+\Delta(r)\left(M-E_{n \kappa}\right) G_{n \kappa}(r)=0
\end{aligned}
$$

On the other hand, the exact spin symmetry occurs when, $\Delta(r)=V(r)-S(r)=0$ and $\Sigma(r)=V(r)+S(r)$ is the q-deformed Eckart potential expressed in equation (18). Therefore for exact spin symmetry, the Dirac equation for the upper spinor expressed in equation (5) becomes

$$
\begin{aligned}
& \left\{\frac{d^{2}}{d r^{2}}-\frac{\kappa(\kappa+1)}{r^{2}}+\frac{2 \kappa}{r} U(r)-U^{2}(r)-\frac{d U}{d r}\right\} F_{n \kappa}(r) \\
& +\left(E_{n \kappa}{ }^{2}-M^{2}\right) F_{n \kappa}(r)-\Sigma(r)\left(E_{n \kappa}+M\right) F_{n \kappa}(r)=0
\end{aligned}
$$

The Dirac equations for both the exact pseudospin symmetry and the exact spin symmetry in equations (8) and (9) are solved using Romanovski polynomials. By solving the Dirac equation for the pseudospin-symmetric case in equation (8), one can obtain the solution of the spinsymmetric case in equation (9) by changing the solution by

$$
\begin{aligned}
& \Delta(r) \rightarrow-\Sigma(r)\left(V_{0} \rightarrow-V_{0} ; V_{1} \rightarrow-V_{1}\right) ; \\
& \frac{d U}{d r} \rightarrow-\frac{d U}{d r} ; E_{p s} \rightarrow-E_{s} \kappa \rightarrow \kappa+1,
\end{aligned}
$$

therefore the Dirac equation is readily solvable. 


\section{Review of q-deformed hyperbolic function}

The q-deformed Eckart potential and the Yukawa-type tensor potential are given as:

$$
\begin{aligned}
& V(r, \theta)=a^{2}\left(V_{0} \frac{e^{-2 r a}}{\left(1-q e^{-2 r a}\right)^{2}}-V_{1} \frac{1+q e^{-2 r a}}{1-q e^{-2 r a}}\right) \\
& U(r)=-V_{2} a^{2} \frac{e^{-r a}}{r}
\end{aligned}
$$

$V_{0}$ and $V_{1}$ represent the depth of the Eckart potential well and are positive. Also, $V_{1}>V_{0}$. Further, $a$ is a positive parameter which controls the width or the range of the potential well; $q>0$ is the deformation parameter of the potential; $V_{2}$ is the strength of the nucleon force; and $a$ is the range of nucleon force [53], while $M$ is the mass of the particle. Here $0<r<\infty$.

The q-deformed hyperbolic potential as introduced by Arai [30] some years ago is defined as

$$
\begin{aligned}
& \sinh _{q} \alpha r=\frac{\left(e^{\alpha r}-q e^{-\alpha r}\right)}{2} ; \cosh { }_{q} \alpha r=\frac{\left(e^{\alpha r}+q e^{-\alpha r}\right)}{2} ; \\
& \tanh _{q} \alpha r=\frac{\sinh _{q} \alpha r}{\cosh _{q} \alpha r} ; \sec h_{q}=\frac{1}{\cosh _{q} \alpha r} ; \\
& \cosh _{q}^{2} \alpha r-\sinh _{q}^{2} \alpha r=q ; 1-\tanh _{q}^{2} \alpha r=q \sec h_{q}^{2} \alpha r
\end{aligned}
$$

By a convenient translation of the spatial variable, one can transform deformed potentials to the corresponding non-deformed ones or vice-versa. By using a translation of spatial variable introduced by Dutra [33]

$$
r \rightarrow r+\frac{\ln \sqrt{q}}{\alpha}
$$

the deformed potential in equation (12) changes into a non-deformed one given as

$$
\begin{aligned}
& \sinh _{q} \alpha r=\sqrt{q} \sinh \alpha r ; \quad \cosh _{q} \alpha r=\sqrt{q} \cosh \alpha r ; \\
& \tanh _{q} \alpha r=\tanh \alpha r \mathrm{z}
\end{aligned}
$$

In the same way, by applying the translation of spatial variable

$$
r \rightarrow r-\frac{\ln \sqrt{q}}{\alpha}
$$

to the non-deformed hyperbolic function potential we obtain the deformed potential as

$$
\sinh \alpha r=\frac{\sinh _{q} \alpha r}{\sqrt{q}} ; \cosh \alpha r=\frac{\cosh _{q} \alpha r}{\sqrt{q}}
$$

The translation of spatial variable in equation (13b) was used to map the energy and wave function of non-deformed potential into deformed forms of modified Poschl-Teller and of hyperbolic Scarf potentials [32]. By applying the concept of hyperbolic functions and the translation of spatial variable, the Dirac equation for q-deformed Eckart potential combined with Yukawa-type tensor potential is expressed in hyperbolic functions.

\section{EXPERIMENTAL METHODS}

For exact spin and pseudospin symmetries, the Dirac equation for q-deformed Eckart potential within the Yukawa type tensor reduces to Schrödinger-type equation. Therefore, it can be solved using Romanovski polynomials. The onedimensional second order differential equation satisfied by Romanovski polynomials is developed based on hypergeometric differential equations. The one-dimensional Schrödinger equation of potential of interest reduces to the differential equation of Romanovski polynomial by appropriate variable and wave function substitutions. The general one-dimensional Schrödinger equation is given as

$$
-\frac{\hbar^{2}}{2 M} \frac{\partial^{2} \Psi(x)}{\partial x^{2}}+V(x) \Psi(x)=E \Psi(x)
$$

where $V(x)$ is an effective potential which is mostly shape-invariant potential, $M$ is the mass of particle, $E$ is the energy eigenvalue of particle. By suitable variable substitution $x=f(s)$ in equation (15) then it changes into generalized hypergeometric type equation expressed as

$$
\frac{\partial^{2} \Psi(s)}{\partial s^{2}}+\frac{\tilde{\tau}(s)}{\sigma(s)} \frac{\partial \Psi(s)}{\partial s}+\frac{\tilde{\sigma}(s)}{\sigma^{2}(s)} \Psi(s)=0
$$

with $\sigma(s)$ and $\tilde{\sigma}(s)$ are mostly polynomials of order two, $\widetilde{\tau}(s)$ is polynomial of order one, s, $\sigma(s)$, $\tilde{\sigma}(s)$, and $\tilde{\tau}(s)$ can have any real or complex values [55]. Equation (16) is solved using variable separation method by introducing new wave function given as

$$
\Psi_{n}(r)=g_{n}(s)=\left(1+s^{2}\right)^{\frac{\beta}{2}} e^{\frac{-\alpha}{2} \tan ^{-1} s} D_{n}^{(\beta, \alpha)}(s)
$$

such that we obtain a hypergeometric type differential equation, which is satisfied by finite Romanovski polynomials $[44,53]$ is expressed as 


$$
\sigma(s) y^{\prime \prime}(s)+\tau(s) y^{\prime}(s)+\lambda y(s)=0
$$

With

$$
\begin{aligned}
& \sigma(s)=a s^{2}+b s+c ; \tau=f s+h ; \\
& -\{n(n-1)+2 n(1-p)\}=\lambda=\lambda_{n}
\end{aligned}
$$

For Romanovski polynomials, the values of parameters in equation (19) are

$$
a=1, b=0, c=1, f=2(1-p) ; h=q^{\prime} ; p>0
$$

therefore by considering equations (19) and (20) we can rewrite equation (18) as

$$
\begin{aligned}
& \left(1+s^{2}\right) \frac{\partial^{2} R_{n}^{\left(p, q^{\prime}\right)}}{\partial s^{2}}+\left\{2 s(-p+1)+q^{\prime}\right\} \frac{\partial R_{n}^{\left(p, q^{\prime}\right)}(s)}{\partial s} \\
& -\{n(n-1)+2 n(1-p)\} R_{n}^{\left(p, q^{\prime}\right)}(s)=0
\end{aligned}
$$

Equation (21) is second order differential equation satisfied by Romanovski polynomials with

$$
y_{n}=R_{n}^{\left(p, q^{\prime}\right)}(s)=D_{n}^{(\beta, \alpha)}(s)
$$

Equation (18) is described in the textbook by Nikiforov-Uvarov [55] where it is cast into self adjoint form and its weight function, $w(s)$, satisfies Pearson differential equation

$$
\frac{d(\sigma(s) w(s))}{d s}=\tau(s) w(s)
$$

The weight function, $w(s)$, is obtained by solving the Pearson differential equation and by applying condition in equations (19) and (20), so we get

$$
w^{\left(p, q^{\prime}\right)}(s)=\left(1+s^{2}\right)^{-p} e^{q^{\prime} \tan ^{-1}(s)}
$$

The corresponding polynomials are classified according to the weight function, and are built up from the Rodrigues representation which is presented as

$$
y_{n}=\frac{B_{n}}{w(s)} \frac{d^{n}}{d s^{n}}\left\{\left(a s^{2}+b s+c\right)^{n} w(s)\right\}
$$

with $B_{n}$ is a normalization constant, and for $\sigma(s)>0$ and $w(s)>0, \quad y_{n}(s)$ 's are normalized polynomials and are orthogonal with respect to the weight function $w(s)$ within a given interval $\left(s_{1}, s_{2}\right)$, which is expressed as

$$
\int_{-\infty}^{\infty} w(s) y_{n}(s) y_{n^{\prime}}(s) d s=\delta_{n n^{\prime}}
$$

This weight function in equation (24) first reported by Routh [41] and then by Romanovski [42]. The polynomial associated with equation (25) are named after Romanovski and will be denoted by $R_{n}^{(p, q)}(s)$. Due to the decrease of the weight function by $s^{-2 p}$, integral of the type

$$
\int_{-\infty}^{\infty} w^{\left(p, q^{\prime}\right)} R_{n}^{\left(p, q^{\prime}\right)}(s) R_{n^{\prime}}^{\left(p, q^{\prime}\right)}(s) d s
$$

will be convergent only if

$$
n^{\prime}+n<2 p-1
$$

This means that only a finite number of Romanovski polynomials are orthogonal, and the orthogonality integral of the polynomial is expressed similar to the equation (26) where $y_{n}=R_{n}^{\left(p, q^{\prime}\right)}(s)$.

The heart of Romanovski polynomials method is in obtaining differential equation that has similar form with equation (21) from onedimensional Schrödinger equation. The Schrödinger equation of the potential of interest will reduce into second order differential equation that is similar to equation (21) by an appropriate transformation of variable, for example, $r=f(s)$, such that generalized hypergeometric equation expressed in equation (16) is obtained. Then equation 16-type is being solved using variable separation method by substituting a new wave function given in equation (17) and finally we get new equation in the form of equation (21) with $\beta$ and $\alpha$ parameters. By comparing equation (21) and new equation, we get the relation between $\beta$ with $p$, and $\alpha$ with $q^{\prime}$. The Romanovski polynomials obtained from Rodrigues formula expressed in equation (25) with the corresponding weight function in equation (24) is expressed as

$$
\begin{aligned}
& R_{n}^{\left(p, q^{\prime}\right)}(s)=D_{n}^{(\beta, \alpha)}(s)=\frac{1}{\left(1+s^{2}\right)^{-p} e^{q^{\prime} \tan ^{-1}(s)}} \\
& \frac{d^{n}}{d s^{n}}\left\{\left(1+s^{2}\right)^{n}\left(1+s^{2}\right)^{-p} e^{q^{\prime} \tan ^{-1}(s)}\right\}
\end{aligned}
$$

If the wave function of the $n^{\text {th }}$ level in equation (17) is rewritten as

$$
\Psi_{n}(r)=\frac{1}{\sqrt{\frac{d f(s)}{d s}}}\left(1+s^{2}\right)^{\frac{-p}{2}} e^{\frac{q^{\prime}}{\tan ^{-1}(s)}} R_{n}^{\left(p, q^{\prime}\right)}(s)
$$


then the orthogonality integral of the wave functions expressed in equation (30) gives rise to orthogonality integral of the finite Romanovski polynomials, that is given as

$$
\int_{0}^{\infty} \Psi_{n}(r) \Psi_{n^{\prime}}(r) d r=\int_{-\infty}^{\infty} w^{\left(p, q^{\prime}\right)} R_{n}^{\left(p, q^{\prime}\right)}(s) R_{n^{\prime}}^{\left(p, q^{\prime}\right)}(s) d s
$$

In this case, the values of $p$ and $q^{\prime}$ are not dependent on the degree of polynomials $n$. However, if either equation (26) or (28) is not fulfilled then the Romanovski polynomials is infinity $[43,45]$. The lower and upper components of Dirac spinors are expressed as wave function in equation (17).

\section{RESULTS AND DISCUSSION}

\section{Exact pseudospin-symmetric case}

By inserting q-deformed Eckart potential and Yukawa-type tensor potential into equation (8) we have

$$
\begin{aligned}
& \left\{\frac{d^{2}}{d r^{2}}-\frac{\kappa(\kappa-1)}{r^{2}}-\frac{2 \kappa}{r} V_{2} a^{2} \frac{e^{-r a}}{r}\right\} G_{n \kappa}(r)+ \\
& \left(-V^{2}{ }_{2} a^{4} \frac{e^{-2 r a}}{r^{2}}+V_{2} a^{3} \frac{e^{-r a}}{r}+V_{2} a^{2} \frac{e^{-r a}}{r^{2}}\right) G_{n \kappa}(r) \\
& -a^{2}\left(\frac{V_{0} e^{-2 r a}}{\left(1-q e^{-2 r a}\right)^{2}}-V_{1} \frac{1+q e^{-2 r a}}{1-q e^{-2 r a}}\right)\left(E_{n \kappa}-M\right) G_{n \kappa}(r) \\
& =-\left(E_{n \kappa}{ }^{2}-M^{2}\right) G_{n \kappa}(r)
\end{aligned}
$$

Equation (32) can not be solved exactly unless we use approximation to the $\frac{1}{r^{2}}$ and $\frac{1}{r}$ terms. For small $a$, or $r a<<1$, the approximation of $\frac{1}{r^{2}}$ has the form of [5]

$$
\begin{aligned}
& \frac{1}{r^{2}} \cong \frac{4 a^{2} e^{-2 a r}}{\left(1-q e^{-2 a r}\right)^{2}} \\
& \frac{1}{r^{2}}=\frac{4 a^{2} e^{-a r}}{\left(1-q e^{-2 a r}\right)^{2}}
\end{aligned}
$$

The two approximations are basically the same since the graphs of both approximations overlap. To obtain the exact solution in equation (32), the $\frac{e^{-r a}}{r^{2}}$ term is substituted by approximation in equation (34), while the $\frac{e^{-2 r a}}{r^{2}}, \frac{e^{-r a}}{r}$, and $\frac{1}{r^{2}}$ terms are substituted by equation (33), and therefore equation (32) becomes

$$
\begin{aligned}
& \left\{\begin{array}{l}
\frac{d^{2}}{d r^{2}}-\frac{4 \kappa(\kappa-1) a^{2} e^{-2 r a}}{\left(1-q e^{-2 a r}\right)^{2}}-\frac{8 \kappa V_{2} a^{4} e^{-2 r a}}{\left(1-q e^{-2 a r}\right)^{2}} \\
-V_{2}^{2} a^{4} e^{-2 r a} \frac{4 a^{2} e^{-2 r a}}{\left(1-q e^{-2 a r}\right)^{2}} \\
+V_{2} a^{3} \frac{2 a e^{-2 r a}}{\left(1-q e^{-2 a r}\right)}+V_{2} a^{2} \frac{4 a^{2} e^{-2 r a}}{\left(1-q e^{-2 a r}\right)^{2}}
\end{array}\right\} G_{n \kappa}(r)- \\
& \left(\frac{V_{0} a^{2} e^{-2 r a}}{\left(1-q e^{-2 r a}\right)^{2}}-V_{1} a^{2} \frac{1+q e^{-2 r a}}{1-q e^{-2 r a}}\right)\left(E_{n \kappa}-M\right) G_{n \kappa}(r) \\
& =-\left(E_{n \kappa}{ }^{2}-M^{2}\right) G_{n \kappa}(r)
\end{aligned}
$$

By applying the definition of q-deformed hyperbolic function in equation (12) and the relation between deformed and non-deformed hyperbolic function in equation (14b), equation (35) is rewritten in terms of hyperbolic functions as

$$
\begin{aligned}
& \left\{\frac{d^{2}}{d r^{2}}-\frac{\kappa(\kappa-1) a^{2}}{\left(\sinh _{q} a r\right)^{2}}-\frac{2 \kappa V_{2} a^{4}}{\left(\sinh _{q} a r\right)^{2}}\right\} G_{n \kappa}(r) \\
& +\left(V_{2} a^{4} \frac{\cosh _{q} a r-\sinh _{q} a r}{\sqrt{q} \sinh _{q} a r}+\frac{V_{2} a^{4}}{\left(\sinh _{q} a r\right)^{2}}\right) G_{n \kappa}(r) \\
& -V_{2}^{2} a^{6} \frac{\left(q+2 \sinh _{q}^{2} a r-2 \sinh _{q} a r \cosh _{q} a r\right)}{q\left(\sinh _{q} a r\right)^{2}} G_{n \kappa}(r) \\
& -a^{2}\left(\frac{V_{0}}{\left(2 \sinh _{q} a r\right)^{2}}-V_{1} \operatorname{coth}_{q} a r\right)\left(E_{n \kappa}-M\right) G_{n \kappa}(r) \\
& =-\left(E_{n \kappa}{ }^{2}-M^{2}\right) G_{n \kappa}(r)
\end{aligned}
$$

By setting

$$
\begin{aligned}
& A_{p s}=\left(\kappa+V_{2} a^{2}-1\right)\left(\kappa+V_{2} a^{2}\right)-V_{0}\left(M-E_{n \kappa}\right) \\
& B_{p s}=\frac{V_{2} a^{2}}{\sqrt{q}}+\frac{2 V_{2}^{2} a^{4}}{q}-V_{1}\left(M-E_{n \kappa}\right) \\
& E^{\prime}=\frac{2 V_{2}^{2} a^{4}}{q}+\frac{V_{2} a^{2}}{\sqrt{q}}-\frac{\left(E_{n \kappa}^{2}-M^{2}\right)}{a^{2}}
\end{aligned}
$$

in equation (36), equation (36) reduces to a onedimensional Schrödinger-type equation

$\left\{\frac{d^{2}}{d r^{2}}-\frac{a^{2} A_{p s}}{\left(\sinh _{q} a r\right)^{2}}+a^{2} B_{p s} \operatorname{coth}_{q} a r\right\} G_{n \kappa}(r)=a^{2} E^{\prime} G_{n \kappa}(r)$

By substitution of spatial variable, $\operatorname{coth}_{q} a r=i x$ in equation (40) we obtain 


$$
\begin{aligned}
& \left(1+x^{2}\right) \frac{d^{2} G_{n \kappa}}{d x^{2}}+2 x \frac{d G_{n \kappa}}{d x} \\
& -\left(q A_{p s}+\frac{q^{2} B_{p s} i x}{1+x^{2}}-\frac{q^{2} E^{\prime}}{1+x^{2}}\right) G_{n \kappa}=0
\end{aligned}
$$

To solve equation (41) we use equation (17) as a new wavefunction,

$$
G_{n \kappa}(x)=g_{n}(x)=\left(1+x^{2}\right)^{\frac{\beta}{2}} e^{\frac{-\alpha}{2} \tan ^{-1} x} D_{n}^{(\beta, \alpha)}(x)
$$

and after manipulating equations (41) and (42) we obtain

$$
\begin{aligned}
& \left(1+x^{2}\right) \frac{\partial^{2} D}{\partial x^{2}}+\{2 x(\beta+1)-\alpha\} \frac{\partial D}{\partial x}- \\
& \left\{\frac{\beta \alpha x-\frac{\alpha^{2}}{4}+\beta^{2}+q^{2} B_{p s} i x-q^{2} E^{\prime}}{1+x^{2}}+q A_{p s}-\beta^{2}-\beta\right\} D=0
\end{aligned}
$$

Equation (43) reduces to differential equation which is satisfied by Romanovski polynomials in equation (21) if the coefficient of the $\frac{1}{1+x^{2}}$ term is set to zero, as follows:

$-\frac{\alpha^{2}}{4}+\beta^{2}-q^{2} E^{\prime}=0$ and $\beta \alpha+q^{2} B_{p s} i=0$

and equation (43) becomes

$$
\left(1+x^{2}\right) \frac{\partial^{2} D}{\partial x^{2}}+\{2 x(\beta+1)-\alpha\} \frac{\partial D}{\partial x}-\left\{q A_{p s}-\beta^{2}-\beta\right\} D=0
$$

By comparing the parameters in equations (21) and (45) we obtain the following relation:

$$
\begin{gathered}
A_{p s}-\beta^{2}-\beta=n(n-1)+2 n(1-p) \\
2(\beta+1)=2(-p+1) \text { and } \alpha=-q^{\prime}
\end{gathered}
$$

From equation (46) we have $p=-\beta$, and since $p>0$, the value of $\beta$ obtained from equation (46) which has physical meaning is

$$
\beta=\beta_{n}=-\sqrt{A_{p s}+\frac{1}{4}}-n-\frac{1}{2}
$$

By using equations (44) and (47) we obtain

$$
\alpha=-\frac{q^{2} B_{p s} i}{\beta}=\frac{q^{2} B_{p s} i}{\sqrt{A_{p s}+\frac{1}{4}}+n+\frac{1}{2}}
$$

The values of $\beta$ obtained from equation (44) is given as

$$
\beta^{2}=\frac{q^{2} E^{\prime} \pm \sqrt{q^{4} E^{\prime 2}-q^{4} B_{p s}^{2}}}{2}
$$

The energy eigenvalues obtained from equations (47) and (49) are

$$
\frac{q^{2} E^{\prime} \pm \sqrt{q^{4} E^{\prime 2}-q^{4} B_{p s}^{2}}}{2}=\left(\sqrt{A_{p s}+\frac{1}{4}}+n+\frac{1}{2}\right)^{2}
$$

that gives

$$
\begin{aligned}
& \left(E_{n \kappa}^{2}-M^{2}\right)=\frac{2 V_{2}^{2} a^{6}}{q}+\frac{V_{2} a^{4}}{\sqrt{q}} \\
& -\frac{a^{2} q^{2} B_{p s}^{2}}{4\left(\sqrt{A_{p s}+\frac{1}{4}}+n+\frac{1}{2}\right)^{2}} \\
& -\frac{a^{2}}{q^{2}}\left(\sqrt{A_{p s}+\frac{1}{4}}+n+\frac{1}{2}\right)^{2}
\end{aligned}
$$

with

$$
A_{p s}=\left(\kappa+V_{2} a^{2}-1\right)\left(\kappa+V_{2} a^{2}\right)-V_{0}\left(M-E_{n \kappa}\right)
$$

and $\quad B_{p s}=\frac{V_{2} a^{2}}{\sqrt{q}}+\frac{2 V_{2}^{2} a^{4}}{q}-V_{1}\left(M-E_{n \kappa}\right)$

The relativistic energy of a nucleon in the field of q-deformed Eckart potential combined with Yukawa-type tensor potential can be obtained using Romanovski polynomials for exact pseudospin symmetry; the result is given in (50). Equation (50) is a transcendental equation. Therefore, the relativistic energy can not be calculated explicitly but it can be calculated numerically using Matlab programming. The energy in equation (50) reduces to the relativistic energy of nucleon in q-deformed Eckart potential field in the absence of Yukawa-type tensor potential when $V_{2}=0$.

To determine the wave function, equations (47) and (48) are inserted into equations (24) and (29) so that we obtain the weight function $w(x)$ and the Romanovski polynomials $R_{n}^{\left(p, q^{\prime}\right)}=R_{n}^{(-\beta,-\alpha)}(x)$ as 


$$
\begin{aligned}
& w^{(-\beta,-\alpha)}=\left(1+x^{2}\right)^{\beta_{n}} e^{-\alpha_{n} \tan ^{-1} x} \\
& =(1-\operatorname{coth} r a)^{\beta_{n}-\frac{i \alpha_{n}}{2}}(1+\operatorname{coth} r a)^{\beta_{n}+\frac{i \alpha_{n}}{2}}
\end{aligned}
$$

And

$$
\begin{aligned}
& R_{n}^{\left(p, q^{\prime}\right)}(x)=R_{n}^{(-\beta,-\alpha)}(r)=\frac{1}{\left(1+x^{2}\right)^{\beta_{n}} e^{-\alpha_{n} \tan ^{-1}(x)}} \\
& \times \frac{d^{n}}{d x^{n}}\left\{\left(1+x^{2}\right)^{\beta_{n}+n} e^{-\alpha_{n} \tan ^{-1}(x)}\right\}
\end{aligned}
$$

where we have applied trigonometric-hyperbolic functions relation given as

$$
\begin{aligned}
& e^{-\frac{\alpha_{n}}{2} \tan ^{-1} x}=e^{-\frac{\alpha_{n}}{2} \tan ^{-1}(-i \operatorname{coth}(a r))} \\
& =\left(\frac{1+\operatorname{coth}(r a)}{1-\operatorname{coth}(r a)}\right)^{\frac{i \alpha_{n}}{4}}
\end{aligned}
$$

where $\beta_{n}$ and $\alpha_{n}$ are as expressed in equations (47) and (48). As a result, the wave function of the $n$th level, which is the lower spinor component of the nucleon, is given by

$$
G_{n \kappa}(f(x))=\left(1+x^{2}\right)^{\frac{\beta_{n}}{2}} e^{\frac{-\alpha_{n}}{2} \tan ^{-1} x} R_{n}^{(-\beta,-\alpha)}(x)
$$

or

$$
\begin{aligned}
& G_{n \kappa}(r)=(1-\operatorname{coth}(r a))^{\frac{\beta_{n}}{2}-\frac{i \alpha_{n}}{4}} \\
& \times(1+\operatorname{coth}(r a))^{\frac{\beta_{n}}{2}+\frac{i \alpha_{n}}{4}} R_{n}^{(-\beta,-\alpha)}(-i \operatorname{coth}(r a))
\end{aligned}
$$

The upper spinor component obtained by using equations (1)-(3) is given as

$$
F_{n \kappa}(r)=\frac{\left(\frac{d}{d r}-\frac{\kappa}{r}+U(r)\right)}{M-E_{n \kappa}} G_{n \kappa}
$$

For this exact pseudospin-symmetric case, the upper spinor component is obtained by using the lower spinor component expressed in equation (54) or (55). Also, it is required that $E_{n \kappa} \neq M$ for equation (56) to exist; this means that under the pseudospin symmetry the nucleon has negative energy.

Since the $\beta_{n}$ and $\alpha_{n}$ parameters, expressed in equations (47) and (48), are dependent on $n$, then the orthogonality of the wave functions may not imply the orthogonality integral of the polynomials [45], that is

$$
\begin{aligned}
& \int_{0}^{\infty} \chi_{n}(r) \chi_{n^{\prime}}(r) d r=\delta_{n n^{\prime}} \\
& \neq \int_{1}^{\infty} w^{(-\beta,-\alpha)} R_{n}^{(-\beta,-\alpha)}(x) R_{n^{\prime}}^{(-\beta,-\alpha)} d x
\end{aligned}
$$

By carrying out the differentiations of equation (52), we find the four lowest unnormalized Romanovski polynomials as

$$
\begin{aligned}
& R_{0}^{\left(-\beta_{0},-\alpha_{0}\right)}(x)=1 \\
& R_{1}^{\left(-\beta_{1},-\alpha_{1}\right)}(x)=\left(\beta_{1}+1\right) 2 x-\alpha_{1} \\
& R_{2}^{\left(-\beta_{2},-\alpha_{2}\right)}(x)=2\left(\beta_{2}+2\right)\left(2 \beta_{2}+3\right) x^{2} \\
& -2 \alpha_{2}\left(2 \beta_{2}+3\right) x+\alpha_{2}^{2}+2 \beta_{2}+4 \\
& R_{3}^{\left(-\beta_{3},-\alpha_{3}\right)}(x)=4 x^{3}\left(\beta_{3}+3\right)\left(2 \beta_{3}+5\right)\left(\beta_{3}+2\right) \\
& -6 \alpha_{3} x^{2}\left(2 \beta_{3}+5\right)\left(\beta_{3}+2\right) \\
& +2 x\left(6 \beta_{3}^{2}+3 \alpha_{3}^{2} \beta_{3}+28 \beta_{3}+6 \alpha_{3}^{2}+34\right) \\
& -2 \alpha_{3}\left(2 \beta_{3}+5\right)-\alpha\left(\alpha^{2}+2 \beta+6\right)
\end{aligned}
$$

The four lowest degrees of unnormalized radial wave functions for arbitrary values of $l$ are calculated by using equations (55) and (58)-(61). It can be seen that the Romanovski polynomials are complex function for odd degrees polynomials but they are real functions for even degrees polynomials.

The three lowest unnormalized wave functions for any $\kappa$ values obtained from equations (55) and (58)-(60) are

$$
\begin{aligned}
& G_{0 \kappa}(r)=(1-\operatorname{coth}(r a))\left\{-\frac{\sqrt{A_{p s}+\frac{1}{4}}+\frac{1}{2}}{2}+q^{2} B_{p s} / 4\left(\sqrt{A_{p s}+\frac{1}{4}}+\frac{1}{2}\right)\right\} \\
& \times(1+\operatorname{coth}(r a))-\left\{\frac{\sqrt{A_{p s}+\frac{1}{4}}+\frac{1}{2}}{2}+q^{2} B_{p s} / 4\left(\sqrt{A_{p s}+\frac{1}{4}}+\frac{1}{2}\right)\right\} \\
& G_{1 \kappa}(r)=(1-\operatorname{coth}(r a))\left\{-\frac{\sqrt{A_{p s}+\frac{1}{4}}+\frac{3}{2}}{2}+q^{2} B_{p s} / 4\left(\sqrt{A_{p s}+\frac{1}{4}}+\frac{3}{2}\right)\right\} \\
& \times(1+\operatorname{coth}(r a))\left\{-\frac{\left.\sqrt{A_{p s}+\frac{1}{4}+\frac{3}{2}}-q^{2} B_{p s} / 4\left(\sqrt{A_{p s}+\frac{1}{4}}+\frac{3}{2}\right)\right\}}{2}\right) \\
& \times\left\{\left(2 \sqrt{\left.\left.A_{p s}+\frac{1}{4}+1\right) i \operatorname{coth} a r-\frac{q^{2} B_{p s} i}{\sqrt{A_{p s}}+\frac{1}{4}+\frac{3}{2}}\right\}}\right.\right.
\end{aligned}
$$




$$
\begin{aligned}
& \left.G_{2 \kappa}(r)=(1-\operatorname{coth}(r a)) \mid-\frac{\sqrt{A_{p s}+\frac{1}{4}}+\frac{5}{2}}{2}+q^{2} B_{p s} / 4\left(\sqrt{A_{p s}+\frac{1}{4}}+\frac{5}{2}\right)\right\} \\
& \left.\times(1+\operatorname{coth}(r a)) \mid-\frac{\sqrt{A_{p s}+\frac{1}{4}}+\frac{5}{2}}{2}-q^{2} B_{p s} / 4\left(\sqrt{A_{p s}+\frac{1}{4}}+\frac{5}{2}\right)\right\} \\
& \times\left\{-\left(4 \sqrt{A_{p s}+\frac{1}{4}}+2\right)\left(\sqrt{A_{p s}+\frac{1}{4}}+1\right) \operatorname{coth}^{2} a r\right. \\
& +\frac{4 q^{2} B_{p s}}{\left(\sqrt{A_{p s}+\frac{1}{4}+\frac{5}{2}}\right)}\left(\sqrt{A_{p s}+\frac{1}{4}}+1\right) \operatorname{coth}^{2} a r \\
& \left.-\left[q^{4} B_{p s}^{2} /\left(\sqrt{A_{p s}+\frac{1}{4}}+\frac{5}{2}\right)^{2}\right]-\left(2 \sqrt{A_{p s}+\frac{1}{4}}+1\right)\right\}
\end{aligned}
$$

where $A_{p s}$ and $B_{p s}$ are as given in equations (37) and (38).

\section{Exact spin-symmetric case}

The Dirac equation of q-deformed Eckart potential with Yukawa-type tensor potential for exact spin symmetry where $\Sigma(r)$ is q-deformed Eckart potential and $U(r)$ is Yukawa-type tensor potential, given as

$$
\begin{aligned}
& \left\{\frac{d^{2}}{d r^{2}}-\frac{\kappa(\kappa+1)}{r^{2}}+\frac{2 \kappa}{r} U(r)-U^{2}(r)-\frac{d U}{d r}\right\} F_{n \kappa}(r) \\
& +\left(E_{n \kappa}^{2}-M^{2}\right) F_{n \kappa}(r)-\Sigma(r)\left(E_{n \kappa}+M\right) F_{n \kappa}(r)=0
\end{aligned}
$$

By inserting q-deformed Eckart potential and Yukawa-type tensor potential in equations (10) and (11) into equation (64) we have

$$
\begin{aligned}
& \left\{\frac{d^{2}}{d r^{2}}-\frac{\kappa(\kappa+1)}{r^{2}}-\frac{2 \kappa}{r} V_{2} a^{2} \frac{e^{-r a}}{r}\right\} F_{n \kappa}(r) \\
& -\left(V^{2}{ }_{2} a^{4} \frac{e^{-2 r a}}{r^{2}}+V_{2} a^{3} \frac{e^{-r a}}{r}+V_{2} a^{2} \frac{e^{-r a}}{r^{2}}\right) F_{n \kappa}(r \\
& -a^{2}\left(V_{0} \frac{e^{-2 r a}}{\left(1-q e^{-2 r a}\right)^{2}}-V_{1} \frac{1+q e^{-2 r a}}{1-q e^{-2 r a}}\right)\left(E_{n \kappa}+M\right) F_{n \kappa}(r) \\
& =-\left(E_{n \kappa}{ }^{2}-M^{2}\right) F_{n \kappa}(r)
\end{aligned}
$$

The solution of equation (66) is worked out similarly to the solution of equation (32). By repeating the steps from equation (33) - (64) we obtain the relativistic energy for exact spin symmetry. For spin symmetry we set

$$
\begin{aligned}
& A_{s}=\left(\kappa+V_{2} a^{2}+1\right)\left(\kappa+V_{2} a^{2}\right)+V_{0}\left(E_{n \kappa}+M\right) \\
& B_{s}=-\frac{V_{2} a^{2}}{\sqrt{q}}+\frac{2 V_{2}^{2} a^{4}}{q}+V_{1}\left(E_{n \kappa}+M\right) \\
& E_{s}{ }^{\prime}=\frac{2 V_{2}^{2} a^{4}}{q}-\frac{V_{2} a^{2}}{\sqrt{q}}-\frac{\left(E_{n \kappa}^{2}-M^{2}\right)}{a^{2}}
\end{aligned}
$$

As expected, the equations for $A_{s}$ and $B_{s}$ are also obtainable from $A_{p s}$ and $B_{p s}$ equations (37) and (38) by applying equation ( $9 a$ ).

By inserting equations (67) - (69) into equation (67) and by changing the exponential function into hyperbolic function we have

$\left\{\frac{d^{2}}{d r^{2}}-\frac{a^{2} A_{s}}{\left(\sinh _{q} a r\right)^{2}}+a^{2} B_{s} \operatorname{coth}_{q} a r\right\} F_{n \kappa}(r)=a^{2} E^{\prime} F_{n \kappa}(r)$

By substitution of variable and wave function respectively, expressed as $\operatorname{coth}_{q} a r=i x$ and

$$
F_{n \kappa}(x)=g_{n}(x)=\left(1+x^{2}\right)^{\frac{\beta_{s}}{2}} e^{\frac{-\alpha_{s}}{2} \tan ^{-1} x} D_{n}^{(\beta, \alpha)}(x)
$$

in equation (70), we obtain

$$
\begin{gathered}
\left(1+x^{2}\right) \frac{\partial^{2} D}{\partial x^{2}}+\{2 x(\beta+1)-\alpha\} \frac{\partial D}{\partial x}- \\
\left\{\frac{\beta \alpha x-\frac{\alpha^{2}}{4}+\beta^{2}+q^{2} B_{s} i x-q^{2} E^{\prime}}{1+x^{2}}+q A_{s}-\beta^{2}-\beta\right\} D=0
\end{gathered}
$$

By comparing equation (72) with equation (21) we obtain the relativistic energy for the nucleon

$$
\begin{aligned}
& \left(E_{n \kappa}^{2}-M^{2}\right)=\frac{2 V_{2}^{2} a^{6}}{q}-\frac{V_{2} a^{4}}{\sqrt{q}}- \\
& \frac{a^{2} q^{2} B_{s}^{2}}{4\left(\sqrt{A_{s}+\frac{1}{4}}+n+\frac{1}{2}\right)^{2}}-\frac{a^{2}}{q^{2}}\left(\sqrt{A_{s}+\frac{1}{4}}+n+\frac{1}{2}\right)^{2}
\end{aligned}
$$

and $D_{n}^{(\beta, \alpha)}=R_{n}^{(-\beta,-\alpha)}(x)$ with the values of $A_{s}$ and $B_{s}$ are expressed in equation (67) and (68).

Without the presence of the Yukawa-type tensor coupling potential, when $V_{2}=0$ and $\kappa(\kappa+1)=l(l+1)$ we get 


$$
\begin{aligned}
& A_{s}=l(l+1)+V_{0}\left(E_{n \kappa}+M\right) \\
& B_{s}=V_{1}\left(E_{n \kappa}+M\right) \\
& E_{s}{ }^{\prime}=-\frac{\left(E_{n \kappa}^{2}-M^{2}\right)}{a^{2}}
\end{aligned}
$$

and equation $(73)$ reduces to

$$
\begin{aligned}
& \left(E_{n \kappa}^{2}-M^{2}\right)=-\frac{a^{2} q^{2} V_{1}^{2}\left(E_{n \kappa}+M\right)^{2}}{4\left(\sqrt{l(l+1)+V_{0}\left(E_{n \kappa}+M\right)+\frac{1}{4}}+n+\frac{1}{2}\right)^{2}} \\
& -\frac{a^{2}}{q^{2}}\left(\sqrt{l(l+1)+V_{0}\left(E_{n \kappa}+M\right)+\frac{1}{4}}+n+\frac{1}{2}\right)^{2}
\end{aligned}
$$

Further, weset $V_{0} \rightarrow \frac{V_{0}}{2 \mu} ; V_{1} \rightarrow \frac{V_{1}}{2 \mu} ; E_{n \kappa}+M=2 \mu$; and $\left(E_{n \kappa}^{2}-M^{2}\right)=2 \mu E_{N R}$ where $E_{N R}=E_{n \kappa}-M$ is the non-relativistic energy of Eckart potential with centrifugal term. Therefore, for $q=1$, the relativistic energy in equation (77) becomes the non-relativistic energy of Eckart potential with centrifugal term as follows

$$
\begin{aligned}
& E_{N R}=-\frac{1}{2 \mu}\left\{q^{2} a^{2}\left(V_{1}^{2}\right) / 4\left(\sqrt{l(l+1)+V_{0}+\frac{1}{4}}+n+\frac{1}{2}\right)^{2}\right. \\
& \left.+\frac{a^{2}}{q^{2}}\left(\sqrt{l(l+1)+V_{0}+\frac{1}{4}}+n+\frac{1}{2}\right)^{2}\right\}
\end{aligned}
$$

The upper spinor components obtained from equation (54), with $A_{s}$ expressed in equation (67) and $B_{s}$ in equation (68), are given as

$$
\begin{gathered}
F_{n \kappa}(f(x))=\left(1+x^{2}\right)^{\frac{\beta_{n}}{2}} e^{\frac{-\alpha_{n}}{2} \tan ^{-1} x} R_{n}^{(-\beta,-\alpha)}(x) \\
R_{n}^{(-\beta,-\alpha)}(x)=\frac{1}{\left(1+x^{2}\right)^{\beta_{n}} e^{-\alpha_{n} \tan ^{-1} x}} \\
\times \frac{d^{n}}{d x^{n}}\left(\left(1+x^{2}\right)^{\beta_{n}+n} e^{-\alpha_{n} \tan ^{-1} x}\right)
\end{gathered}
$$

With

$$
\begin{aligned}
& \beta_{n}=\beta_{s n}=-\sqrt{A_{s}+\frac{1}{4}}-n-\frac{1}{2} \\
& \alpha_{n}=\frac{q^{2} B_{s} i}{\sqrt{A_{s}+\frac{1}{4}}+n+\frac{1}{2}}
\end{aligned}
$$

By using equations (67) and (68) together with equations (62)-(64) we obtain the Romanovski polynomials for the three lowest upper components of Dirac spinors. The lowest spinor component for exact spin symmetric case is obtained from equation (79) as

$$
G_{n \kappa}(r)=\frac{\left(\frac{d}{d r}+\frac{\kappa}{r}-U(r)\right)}{M+E_{n \kappa}} F_{n \kappa}
$$

with $E_{n \kappa} \neq-M$. This condition leads to the relativistic energy of the nucleon for exact spin symmetry being always positive.

\section{CONCLUSION}

The Dirac equation for q-deformed Eckart potential with Yukawa-type tensor potential is solved exactly using Romanovski polynomial within the approximation scheme proposed by Greene and Aldrich. There are two solution types: exact pseudospin symmetry and exact spin symmetry. For the exact pseudospin symmetry solution, we directly obtain the lower spinor component and find that relativistic energy of the nucleon is always negative. In this case, the upper spinor component of moving nucleon is found from the lower one. For the exact spin symmetry solution, the upper spinor component is obtained directly from the Dirac equation. It is found that the relativistic energy of the moving nucleon is always positive. The difference between the lower spinor component for pseudospinsymmetric case and the upper spinor component for spin-symmetric case is on the values of $A$ and $B$ parameters in both Dirac equations. Without the presence of the tensor potential for exact spinsymmetric case, the relativistic energy of the system reduces to the non-relativistic energy of q-deformed Eckart potential with centrifugal term in the nonrelativistic limit.

\section{ACKNOWLEDGMENT}

This work is partially supported by the Hibah DIKTI, grant No. 165/UN27.11/PN2013.

\section{REFERENCES}

1. M. Hamzavi and A.A. Rajabi, Adv. H. En. Phys. 2013 (2013) 12.

2. S.M. Ikhdair and R. Sever, arXiv: $1001.4327 \mathrm{v} 2$ [math-ph] (2010) 28. 
3. M. Eshghi, J. Sci. Res. 3 (2011) 493.

4. A.N. Ikot, Commun. Theor. Phys. 59 (2013) 268.

5. O. Aydogdu, E. Maghsoodhi and H. Hassanabadi, Chin. Phys. B22 (2013) 010302.

6. Z. Ai-Ping and Q. Wen-Chao, High Energy Phys. and Nucl. Phys. 31 (2007) 1027.

7. C.H. Chen, Phys. Lett. A 339 (2005) 283.

8. O. Aydogdu and R. Sever, Phys. Lett. B. 703 (2011) 379 .

9. A. Soylu, O. Bayrak and I. Boztosun, J. Math. Phys. 48 (2007) 082302.

10. R.L. Greene and C. Aldrich, Phys. Rev. A 14 (1976) 2363.

11. A.A. Smorodinsky and K.H. Valiev, Nuovo Cimento A 52 (1967) 1061.

12. Z.M. Chang and W.Z. Bang, Chin. Phys. 16 (2007) 1863.

13. W.C. Qing, Chin. Phys. 12 (2003) 136.

14. B. Gönül and I. Zorba, Phys. Lett. A 269 (2000) 83.

15. F. Yasuk, C. Berkdemir and A. Berkdemir, J. Phys. A: Math. Gen. 38 (2005) 6579.

16. C.Y. Chen and S.H. Dong, Phys. Lett. A 335 (2005) 374.

17. C.Y. Chen, Phys. Lett. A 339 (2005) 283.

18. C.Y. Chen, C.L. Liu and D.S. Sun, Phys. Lett. A 305 (2002) 341.

19. K.T. Hecht and A. Adler, Nucl. Phys. A 137 (1969) 129.

20. A. Arima, M. Harvey and K. Shimizu, Phys. Lett. B 30 (1969) 517.

21. J.N. Ginocchio, Phys. Rep. C 69 (2004) 034313.

22. J.N. Ginocchio, Phys. Rev. Lett. 78 (1997) 436.

23. J. Meng, K. Sugaware-Tanabe, S. Yamaji and A. Arima, Phys. Rev. C 59 (1999) 154.

24. J. Dudek, W. Nazarewicz, Z. Szymanski and G.A. Leander, Phys. Rev. Lett. 59 (1987) 1405.

25. D. Troltenier, C. Bahri and J.P. Draayer, Nucl. Phys. A 586 (1995) 53.

26. P.R. Page, T. Goldman and J.N. Ginocchio, Phys. Rev. Lett. 86 (2001) 204.

27. J.N. Ginocchio, Phys. Rep. 414 (2005) 165.
28. K.D. Sviratcheva, C. Bahri, A.I. Georgieva and J.P. Draayer, Phys. Rev. Lett. 93 (2004) 152501.

29. P.N. Swamy, Int. J. Mod. Phys. B12 (1998) 32.

30. A. Arai, J. Math. An. App. 158 (1991) 63.

31. H. Eğrifes, D. Demirhan and F. Büyükkilic, Phys. Lett. A 275 (2000) 229.

32. A. Suparmi, C. Cari and H. Yuliani, Adv. Phys. Theor. App. 16 (2013).

33. A.S. Dutra, arXiv:quant-ph/ 0501094v1 (2005).

34. S.M. Ikhdair, J. Math. Phys. 52 (2011) 22.

35. C. Eckart, Phys. Rev. 35 (1930) 1303.

36. M. Azizi, N. Salehi and A.A. Rajabi, ISRN High Energy Physics 2013 (2013) 310392.

37. H. Yukawa, Proc. Phys. Math. Soc. Jpn. 17 (1935) 48.

38. H. Yukawa, Proc. Phys.Math. Soc. Jpn. 17 (1935) 48.

39. H. Yukawa and S. Sakara, S. Proc. Phys. Math. Soc. Jpn. 19 (1937) 1084.

40. W. Koepf and M. Masjed-Jamei, Integral Transform and Special Functions 17 (2006) 559.

41. E.J. Routh, Proc. London Math. Soc.16 (1884) 245.

42. V.I. Romanovski, Compt. Rend. Ac. Sci. Paris 188 (1929) 1023.

43. A. Raposo, H.J. Weber, D.E. Alvarez-Castillo and M. Kirchbach, C. Eur. Phys. J. 5 (2007) 253.

44. D.E. Alvarez-Castillo and M. Kirbach, Rev. Mex. Fis. E 53 (2007) 143.

45. D.E. Alvarez-Castillo, C.B. Compean and M. Kirbach, arXiv: 1105. 1354v1 (quant-ph] (2011).

46. C.B. Compean and M. Kirbach, arXiv:nuclth/0610001v1 (2006) 1.

47. R. Lisboa, M. Malheiro, A.S. de Castro, P. Alberto and M. Fiolhais, Phys. Rev. C.69 (2004) 024319.

48. H. Hassanabadi, E. Maghsoodi and S. Zarrinkamar, Commun. Theor. Phys. 58 (2012) 807.

49. A Soylu, O Bayrak and I. Boztosun, J. Phys. A: Math. Theor. 41 (2008) 065308.

50. S.M. Ikhdair, J. Math. Phys. 51 (2010) 023525. 
51. C.S. Jia, T. Chen and L.G. Cui, Phys. Lett. A. 373 (2009) 11621.

52. X.Q. Luo and H. Kroger, Sci. China Ser. G.49 (2006) 60.
53. A.F. Nikiforov and V.B. Uvarov, Special Functions of Mathematical Physics, Birkhauser, Verlag, Basel (1988) 4. 